\title{
Factores que influyen en el cumplimiento del pago del Impuesto al Valor del Patrimonio Predial en la ciudad de Puno-Perú
}

\author{
Julio Cesar Quispe Mamani \\ jcquispe@unap.edu.pe \\ Marcial Guevara Mamani \\ mguevara@unap.edu.pe \\ Cesar Elías Roque Guizada \\ cerroguis@hotmail.com \\ Hugo Ruben Marca Maquera \\ hugomarcaunam@gmail.com \\ Victor Rogelio Marca Maquera \\ vickroy-@hotmail.com
}

\section{RESUMEN}

El objetivo de la investigación fue determinar los factores que influyen en el cumplimiento del pago del Impuesto al Valor del Patrimonio Predial, siendo un tema muy relevante para ciudad de Puno, para lo cual se utilizó el modelo econométrico Logit Binomial. La población del presente estudio está compuesta por los contribuyentes de la ciudad de Puno que fue obtenida de la página web del municipio de Puno, por lo que la muestra ascendió a 375 contribuyentes, considerando las variables cultura tributaria que es medido por los indicadores de conciencia, educación y difusión tributaria; también se midió el nivel educativo y el ingreso familiar mensual. Se obtuvo como resultado que, los factores que influyen en el cumplimiento del pago del Impuesto al Valor del Patrimonio Predial en la ciudad de Puno son la cultura tributaria con $22.77 \%$, nivel educativo con $1.86 \%$ y el ingreso familiar mensual del contribuyente con una probabilidad de $10.95 \%$.

Palabras clave: Impuesto al Valor del Patrimonio Predial, cultura tributaria, cumplimiento, ingreso familiar mensual, modelo logit binomial, nivel educativo. 


\title{
Factors that influence the payment of the Property Value Tax in the city of Puno-Peru
}

\begin{abstract}
The objective of the investigation was to determine the factors that influence the fulfillment of the payment of the Tax on the Property Property Value, being a very relevant topic for the city of Puno, for which the Logit Binomial econometric model was used. The population of this study is made up of taxpayers from the city of Puno, which was obtained from the website of the municipality of Puno, so the sample amounted to 375 taxpayers, considering the variables of tax culture that is measured by the indicators of conscience. , tax education and dissemination; Educational level and monthly family income were also measured. It was obtained as a result that the factors that influence compliance with the payment of the Property Tax Value in the city of Puno are the tax culture with $22.77 \%$, educational level with $1.86 \%$ and the taxpayer's monthly family income with a probability $10.95 \%$.
\end{abstract}

Key word: Property Equity Value Tax, tax culture, compliance, monthly family income, binomial logit model, educational level.

Artículo recibido: 01. agosto. 2020 Aceptado para publicación: 12. setiembre. 2020 Correspondencia: jcquispe@ unap.edu.pe Conflictos de Interés: Ninguna que declarar 


\section{INTRODUCCIÓN}

Cultura tributaria se define como la conducta manifestada en el cumplimiento permanente de los deberes tributarios con base en la razón, la confianza y la afirmación de los valores de ética personal, respeto a la ley, responsabilidad ciudadana y solidaridad social de todos (Agurto, 2018). Definido también como el conjunto de conocimientos, valoraciones y actitudes referidas a los tributos, así como al nivel de conciencia respecto de los deberes y derechos que derivan para los sujetos activos y pasivos de esa relación tributaria (Perdomo \& de Egáñez, 2015). Por consiguiente, podemos considerar que la cultura tributaria es el nivel de conocimiento que tienen los individuos de una sociedad acerca del sistema tributario y sus funciones (Flores, 2016). Es necesario que todos los ciudadanos de un país posean una fuerte Cultura Tributaria para que puedan comprender que los tributos son recursos que recauda el Estado para realizar obras orientadas al bien común (Urteaga, Serrano \& Antonio, 2019).

Además, el nivel educativo viene cobrando mayor relevancia para el cumplimiento de las obligaciones tributarias de la población con el estado, es por lo que esta variable cumple la función formar e informar a la población sobre los deberes que se asume desde la edad joven, en el primer caso ayuda a determinar él porque y el para qué es importante cumplir con las obligaciones con el estado y el segundo tiene relación con el que y el cómo entender sobre las obligaciones con el estado y los derechos tributarios (Vílchez-Román Rojas-Mendoza \& Huapaya-Huapaya, 2020). De ahí que se debe de cumplir informando y formando buenos contribuyentes a través de la sensibilización y la socialización, integrando la conducta social ligada al cumplimiento de las normas vigentes que se tiene en la sociedad donde viven (Zapata \& Muñoz-Mora (2013).

Complementariamente el ingreso mensual familiar como variable económica tiene relevancia en la toma de decisiones para cumplir con las obligaciones con el estado (Mamani, Guevara, Ccoa \& Ramirez, 2019). En este sentido, la economía familiar y su comportamiento en la sociedad, considerando el precio de los bienes y servicios que adquiere, el consumo, el ahorro y el empleo que esta tiene ya sea de manera formal e informal evidenciaran sus condiciones económicas como familia (Muñoz \& Ruano, 2010). 
Por otro lado, la función primordial de las municipalidades es suministrar y equilibrar una adecuada prestación de los servicios públicos, para lograr el desarrollo sostenible de su respectiva jurisdicción, con el objetivo de mejorar la calidad de vida de los ciudadanos. Por ende, las municipalidades para dar cumplimiento a sus funciones requieren de ingresos, y son los ciudadanos quienes financian a la municipalidad, a través del pago de los impuestos, sobre todo del Impuesto al Valor del Patrimonio Predial (Mamani, Guevara, Ccoa, \& Ramirez, 2019), puesto que representa la principal fuente de ingresos económicos y permite disponer de ellos para los gastos de la entidad local y para la realización de proyectos de inversión (Miranda, 2019).

La Municipalidad Provincial de Puno es la encargada de la recaudación, administración y fiscalización del Impuesto al Valor del Patrimonio Predial de los inmuebles ubicados en la Provincia de Puno (Mamani, 2017). Este tributo grava el valor de los predios urbanos y rústicos en base a su autovalúo (Altamirano, Oblitas \& Ramiro, 2018), la misma que se obtiene aplicando los aranceles y precios unitarios de construcción que formula el Consejo Nacional de Tasaciones y aprueba el Ministerio de Vivienda, Construcción y Saneamiento todos los años (Zegovia \& Manuel, 2016).

Por lo tanto, los gobiernos Municipales deben utilizar estrategias para informar a la población y obtenga conocimientos sobre el tema y comprenda la importancia de sus responsabilidades tributarias. En algunos casos los contribuyentes desconocen cuál es el destino de los recursos y poseen una idea errónea sobre la utilización de dichos recursos.

Del cual el problema es que en la actualidad existe un incumplimiento en el pago del Impuesto al Valor del Patrimonio Predial por parte de los contribuyentes de la ciudad de Puno, se aprecia que la mayor parte de la población no realizan sus pagos en las fechas programadas, incluso creen que estos son utilizados para beneficio personal y no para el Distrito, la municipalidad provincial no utiliza ninguna estrategia de difusión tributaria para concientizar y dar a conocer la importancia del pago de sus tributos, para mejorar el incremento de dicho impuesto; es por ello que las preguntas de investigación son: ¿Cuál es la influencia de la cultura tributaria, el ingreso familiar mensual y nivel educativo en el cumplimiento de pago del Impuesto al Valor del Patrimonio Predial?. 
Por lo tanto, el objetivo de la presente investigación es determinar los factores que influyen en el cumplimiento del pago del Impuesto al Valor del Patrimonio Predial en la ciudad de Puno. Además, la hipótesis de investigación es que los factores que influyen en el cumplimiento del pago del Impuesto al Valor del Patrimonio Predial en la ciudad de Puno son la cultura tributaria, nivel educativo y el ingreso familiar mensual del contribuyente.

\section{2- MATERIALES Y MÉTODOS}

\section{Diseño metodológico}

El diseño de investigación es no experimental, de tipo de transversal correlacional, puesto que implica recolectar datos en un solo momento, a fin de describir variables y analizar su influencia e interrelación (Sampieri, Collado, \& Lucio, 2010).

\section{Métodos}

Corresponde al método analítico y deductivo, toda vez que se observó el comportamiento y la actitud de los propietarios de predios respecto al pago del Impuesto al Valor del Patrimonio Predial.

\section{Enfoque}

El enfoque es cuantitativo-cualitativo; por la razón de que se manipularan los datos de las variables a conveniencia de la investigación, ya que se van recopilar datos por medio del instrumento de encuesta a los contribuyentes. Se analizó mediante un modelo econométrico donde se aplicó el método de estimación (Agresti, 2007).

\section{Técnicas}

Se utilizó un cuestionario de encuesta, a fin de indagación, exploración y recolección de datos mediante preguntas formuladas de manera directa o indirectamente a los sujetos que constituyen la unidad de análisis.

\section{Análisis de variables}

La descripción de las variables en consideración en la presente investigación son las que se muestran en la tabla 1, donde la variable dependiente es dicotómica (Binaria): 
Tabla 1. Análisis de variables de la investigación

\begin{tabular}{|c|c|c|c|c|c|c|}
\hline Variables & Factor & Indicador & Categoría & $\begin{array}{c}\text { Tipo de } \\
\text { dato }\end{array}$ & $\begin{array}{l}\text { Escala de } \\
\text { medición }\end{array}$ & $\begin{array}{l}\text { Instrumento } \\
\text { recolección }\end{array}$ \\
\hline \multicolumn{7}{|l|}{ Dependiente } \\
\hline $\begin{array}{l}\text { Cumplimiento del } \\
\text { Impuesto al Valor } \\
\text { del Patrimonio } \\
\text { Predial }\end{array}$ & Social & & $\begin{array}{l}\text { No cumple } \\
\text { Si cumple }\end{array}$ & Cualitativo & Nominal & \multirow{5}{*}{ 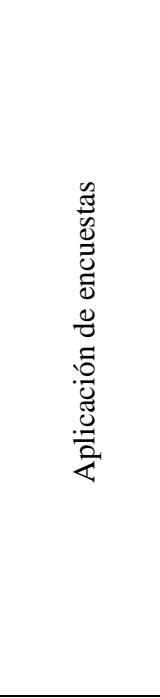 } \\
\hline Independientes & & & & & & \\
\hline Cultura tributaria & Cultural & $\begin{array}{l}\text { Conciencia tributaria } \\
\text { Educación tributaria } \\
\text { Publicidad y } \\
\text { difusión }\end{array}$ & $\begin{array}{l}\text { Si tiene cultura tributaria } \\
\text { No tiene cultura tributaria }\end{array}$ & Cualitativo & Nominal & \\
\hline Ingreso & Económico & $\begin{array}{l}\text { Ingreso familiar } \\
\text { mensual }\end{array}$ & $\begin{array}{l}\text { Menor a s/. } 400.00 \\
\text { De } s / .401 .00-s / .900 .00 \\
\text { De } S / .901 .00-S / .1400 .00 \\
\text { De } S / .1401 .00-S / .1800 .00 \\
\text { De } S / .1801 .00-S / .2200 .00 \\
\text { De } S / .2201 .00-S / .2700 .00\end{array}$ & Cuantitativo & Nominal & \\
\hline Nivel educativo & Cultural & $\begin{array}{l}\text { Nivel educativo del } \\
\text { contribuyente }\end{array}$ & $\begin{array}{l}\text { Sin instrucción } \\
\text { Primaria } \\
\text { Secundaria } \\
\text { Superior }\end{array}$ & Cualitativo & Nominal & \\
\hline
\end{tabular}

\section{Población}

Para efectos del estudio, se consideró una población de 20,300 contribuyentes que realizaron pagos en el 2019, ya sean propietarios de uno a más predio.

\section{Muestra}

La selección de la muestra para este trabajo de investigación se ha empleado el muestreo probabilístico, específicamente el muestreo aleatorio simple, toda vez que, cada elemento de la población tiene la misma probabilidad de ser seleccionado para pertenecer a la muestra. Para lo cual utilizó la siguiente formula:

$$
n=\frac{Z^{2} N p q}{E^{2}(N-1)+Z^{2} p q}
$$

Dónde:

$\mathrm{n}=$ Tamaño de muestra (número de encuestados)

$\mathrm{Z}=$ Valor de distribución normal según él nivel de confianza deseado

$\mathrm{p}=$ Proporción de propietarios que cumplen con el pago de Impuesto al Valor del Patrimonio Predial.

$\mathrm{q}=$ Proporción de propietarios que no cumplen con el pago del Impuesto al Valor del Patrimonio Predial (1-p). 
$\mathrm{N}=$ número de contribuyentes del Impuesto al Valor del Patrimonio Predial.

$\mathrm{E}=$ margen de error

Reemplazando los datos en la fórmula para hallar la muestra se tiene lo siguiente:

$$
\begin{gathered}
n=\frac{\left(1.96^{2}\right)(20300)(0.47)(0.53)}{(0.05)^{2}(20300-1)+(1.96)^{2}(0.5)(0.5)} \\
n=\frac{19425.93}{51.7044} \\
n=375.41 \\
\mathrm{n}=375
\end{gathered}
$$

\section{RESULTADOS Y DISCUSIÓN}

\section{Análisis descriptivo de las variables}

En esta sección se muestra los resultados obtenidos según la hipótesis, de acuerdo a las encuestas realizadas que fueron un total de 375 contribuyentes, explicando el comportamiento de las variables que posee el modelo logit binomial. El comportamiento del contribuyente sobre los distintos escenarios está sujeto a distintos factores que le permiten tomar una decisión acorde a la realidad a la que se encuentra de ahí que su análisis es determinante para evidenciar las dificultades que estas enfrentan en el proceso de contribución del impuesto.

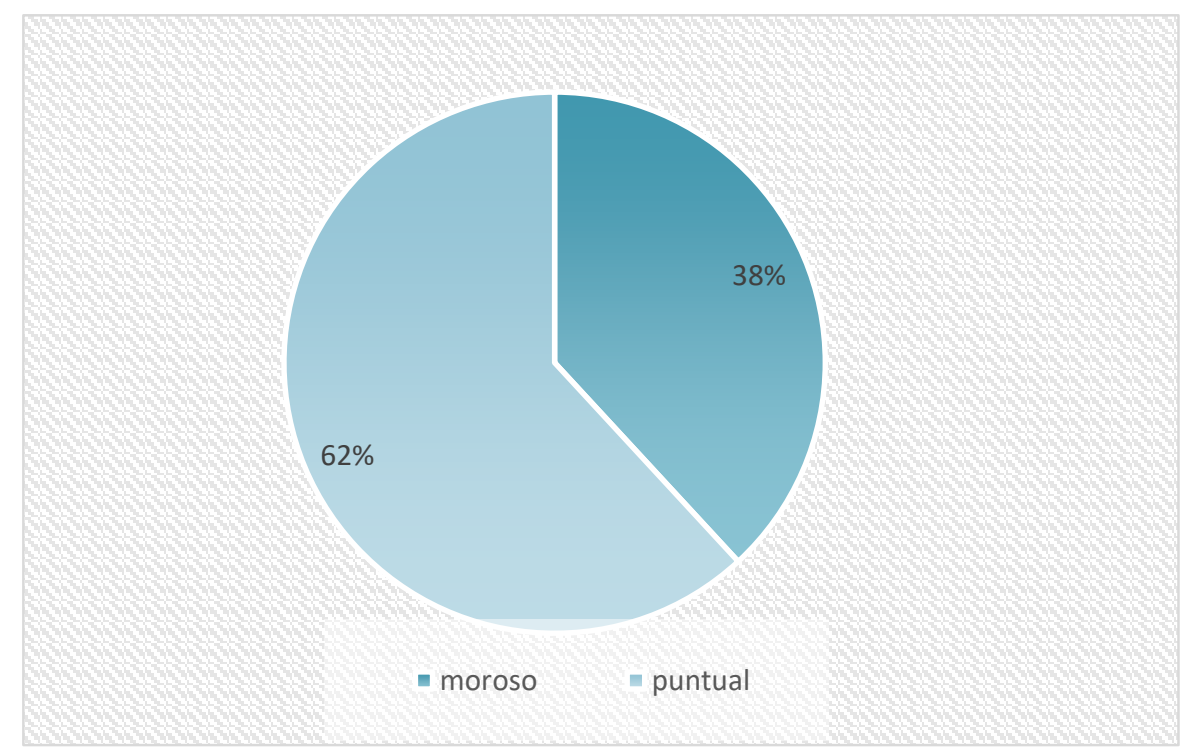

Figura 1. Estado situacional del contribuyente 
Considerando el comportamiento de la población objeto de estudio, al considerar el análisis del estado situacional del contribuyente, el 62\% (243 personas) son puntuales con el pago del Impuesto al Valor del Patrimonio Predial y el 38\% (142 personas) son morosas, reflejando que existe una alta tasa de incumplimiento en los pago del impuesto en análisis, esto dado por el destiempo o con retraso que se realiza (figura 1).

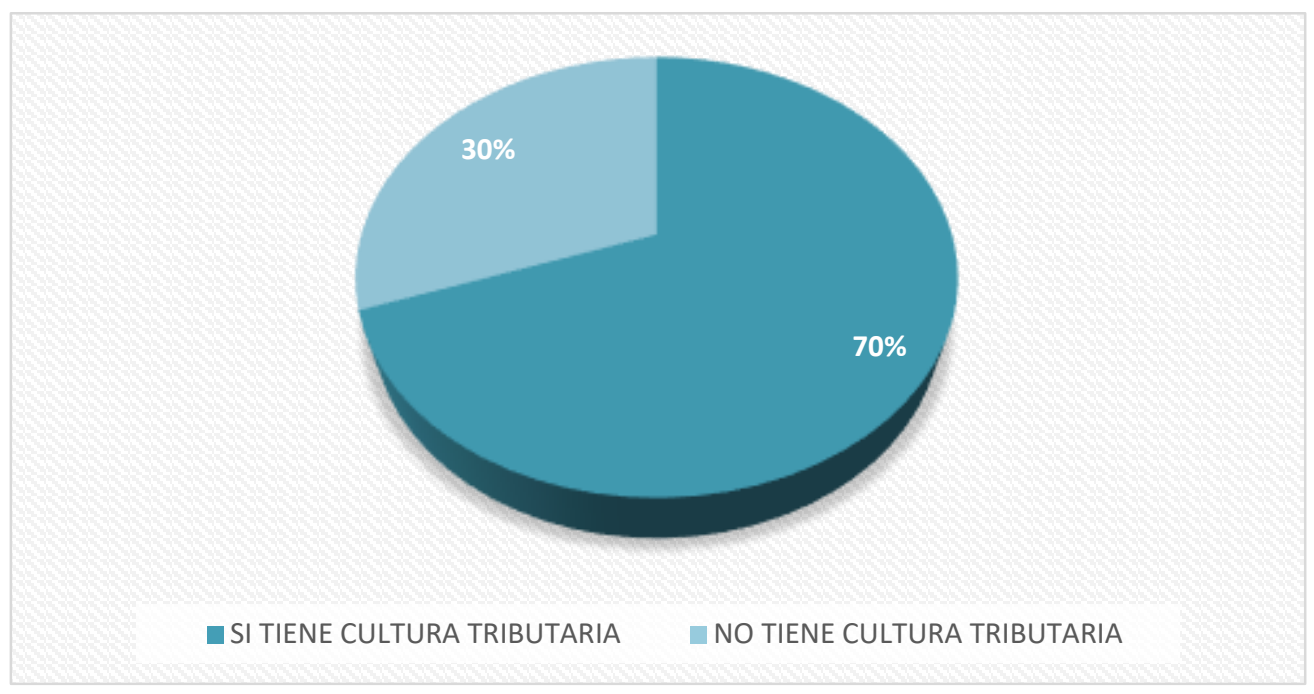

Figura 2. Cultura tributaria del contribuyente

En el ámbito de estudio, al considerar la situación de la cultura tributaria se encontró que, de los 375 contribuyentes encuestados, el 70\% (261 personas) si poseen cultura tributaria y el 30\% (114 personas) no tiene cultura tributaria (Figura 2). Por lo que, al analizar los indicadores de la cultura tributaria como son: la conciencia, educación y difusión tributaria explicadas en los siguientes gráficos, se muestra que la parte de los contribuyentes tienen conciencia y educación tributaria, así mismo se muestra que la difusión tributaria presentada por la municipalidad es ineficiente.

Realizando un análisis especifico a los indicadores que explican a la cultura tributaria, la conciencia tributaria del contribuyente es resaltante, toda vez que, de los 375 contribuyentes se puede apreciar que $271(72 \%)$ encuestados son conscientes de pagar el Impuesto al Valor del Patrimonio Predial a la municipalidad provincial de Puno, y solo el $104(28 \%)$ de los encuestados no son conscientes respecto al tema en cuestión, 
reflejando en este sentido la importancia que tiene la educación tributaria en las familias (Figura 3).

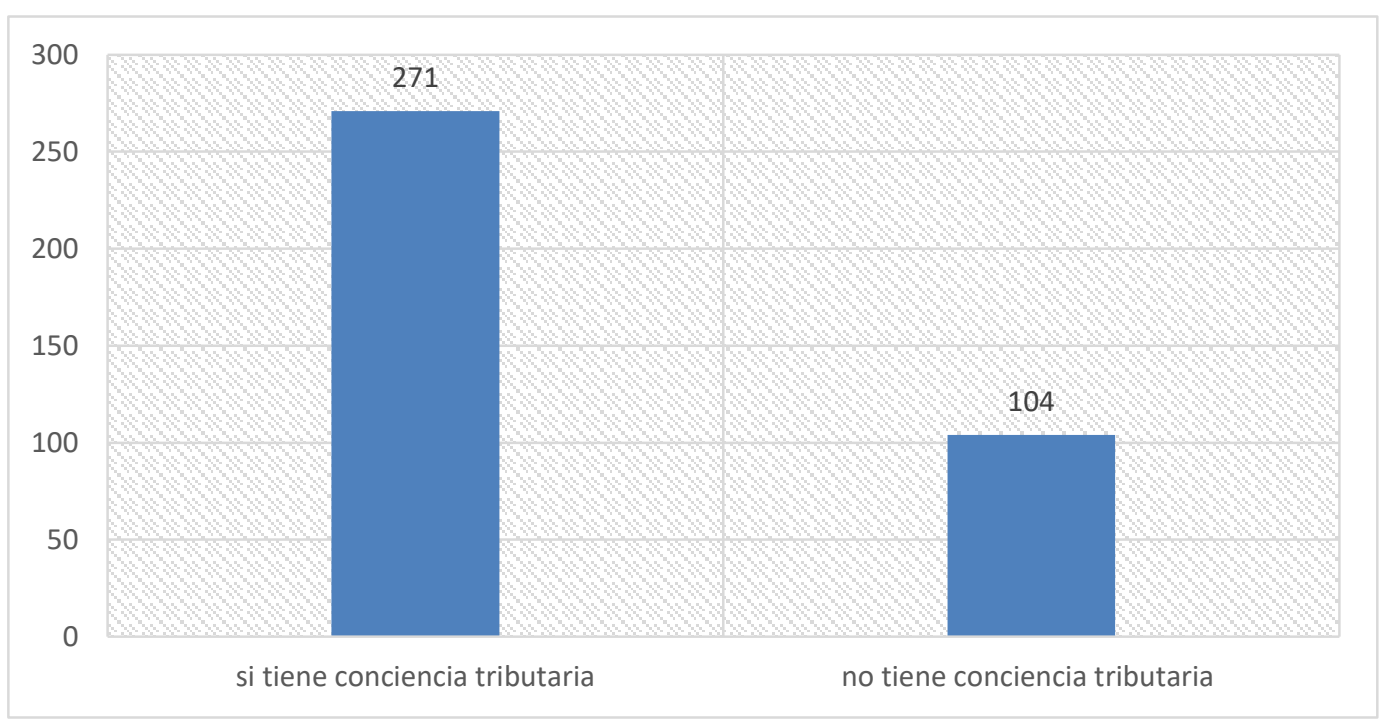

Figura 3. Conciencia tributaria del contribuyente.

Por ende, se puede determinar que la conciencia tributaria que tiene los contribuyentes es complementada por el nivel de educación que estas perciben traducido en la cantidad de años que recibieron educación, pero con mayor especificidad la educación tributaria. Al consultar sobre la misma, del total de encuestados, el 256 (68\%) manifestaron que, si tienen conocimiento sobre las tasas de contribución municipales, y solo el 119 (32\%) no tienen conocimiento acerca del tema (Figura 4).

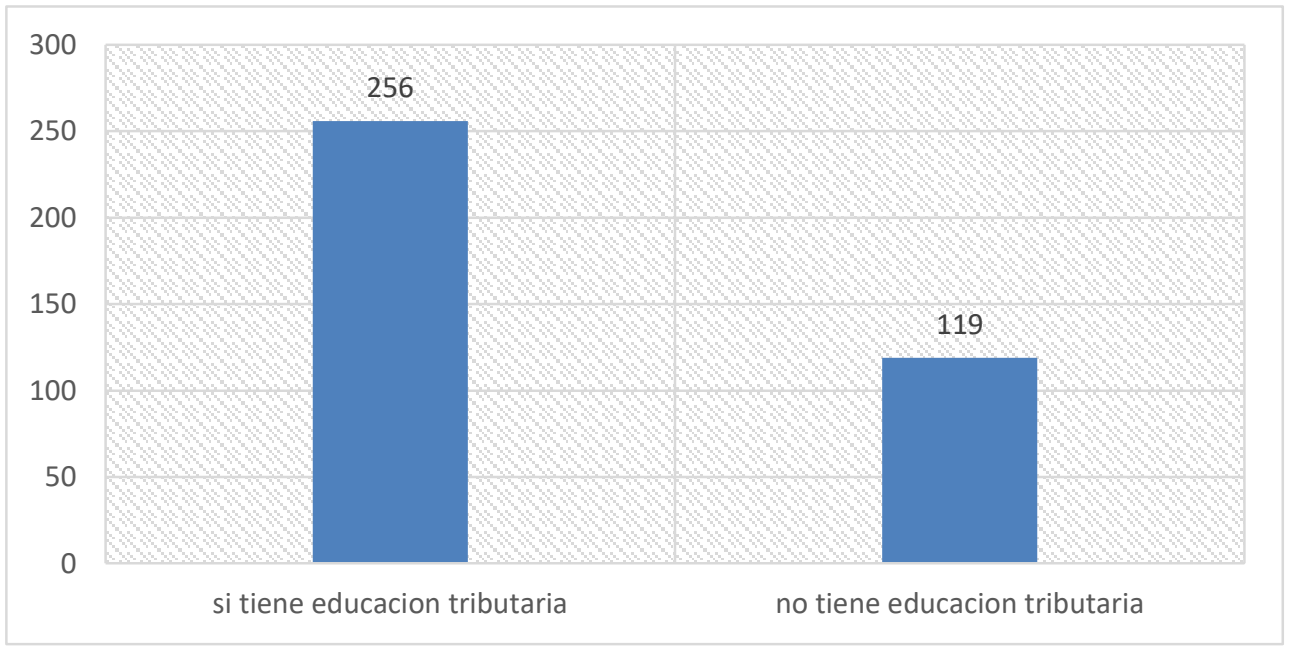

Figura 4. Educación tributaria del contribuyente 
El dilema del contribuyente es cuando realizar y donde realizar el pago de dichos impuestos, por lo que la labor que cumplen las oficinas encargadas sobre el tema de difusión sobre el cumplimiento de las obligaciones tributarias es determinante; en este sentido, al consultar sobre este tema, del total de encuestados, 295 (el 79\%) expresaron que mediante la municipalidad de Puno no recibieron charlas sobre tributación y 80 (el $21 \%$ ) recibieron charlas, viendo así que la falta de difusión se observa claramente como un problema frente al cumplimiento del pago del Impuesto al Valor del Patrimonio Predial (Figura 5).

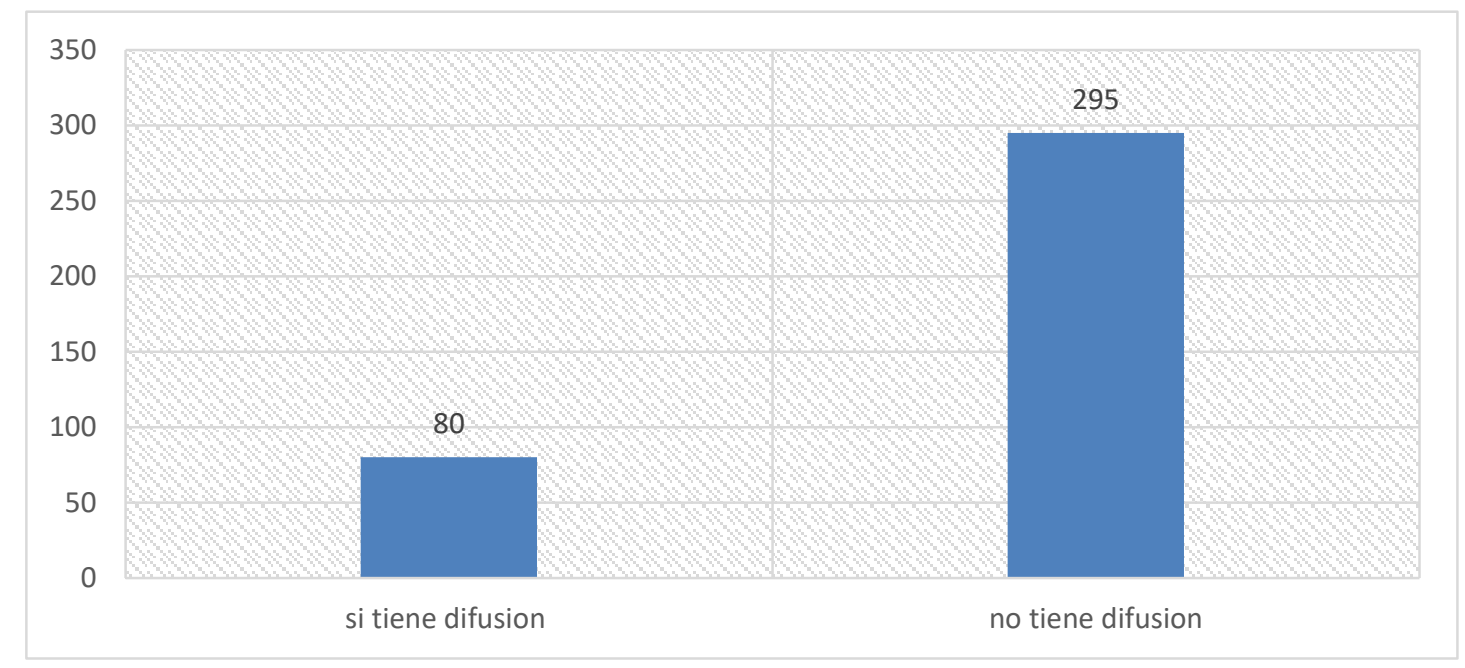

Figura 5. Difusión sobre cumplimiento de obligaciones tributarias

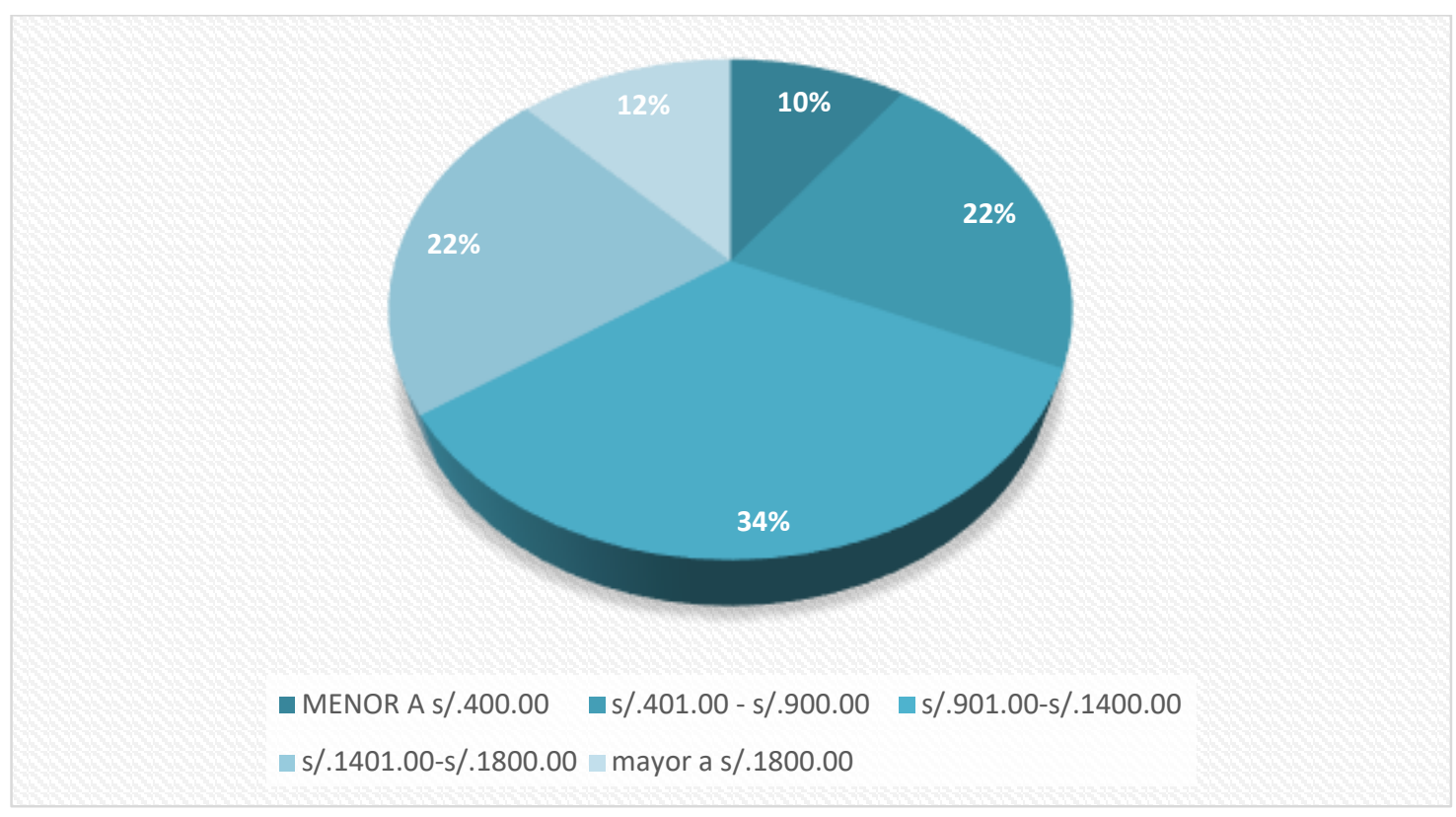

Figura 6. Ingreso familiar mensual del contribuyente. 
Por otro lado, quizá la variable determinante que es resaltante es el ingreso familiar mensual de los contribuyentes, las mismas que tienen diferentes fuentes, ya sea de la actividad económica o de servicios. En este sentido, al consultar al encuestado sobre la misma, el $10 \%$ percibe un sueldo menor a S/ 400 soles, el 22\%, entre S/ 400 a S/ 900 soles, el $34 \%$ entre S/900 - S/1,400 soles, mientras que el $22 \%$ de S/ 1,400 a S/ 1,800 soles y el $12 \%$ restante percibe un salario mayor a $\mathrm{S} / 1,800$ soles (Figura 6).

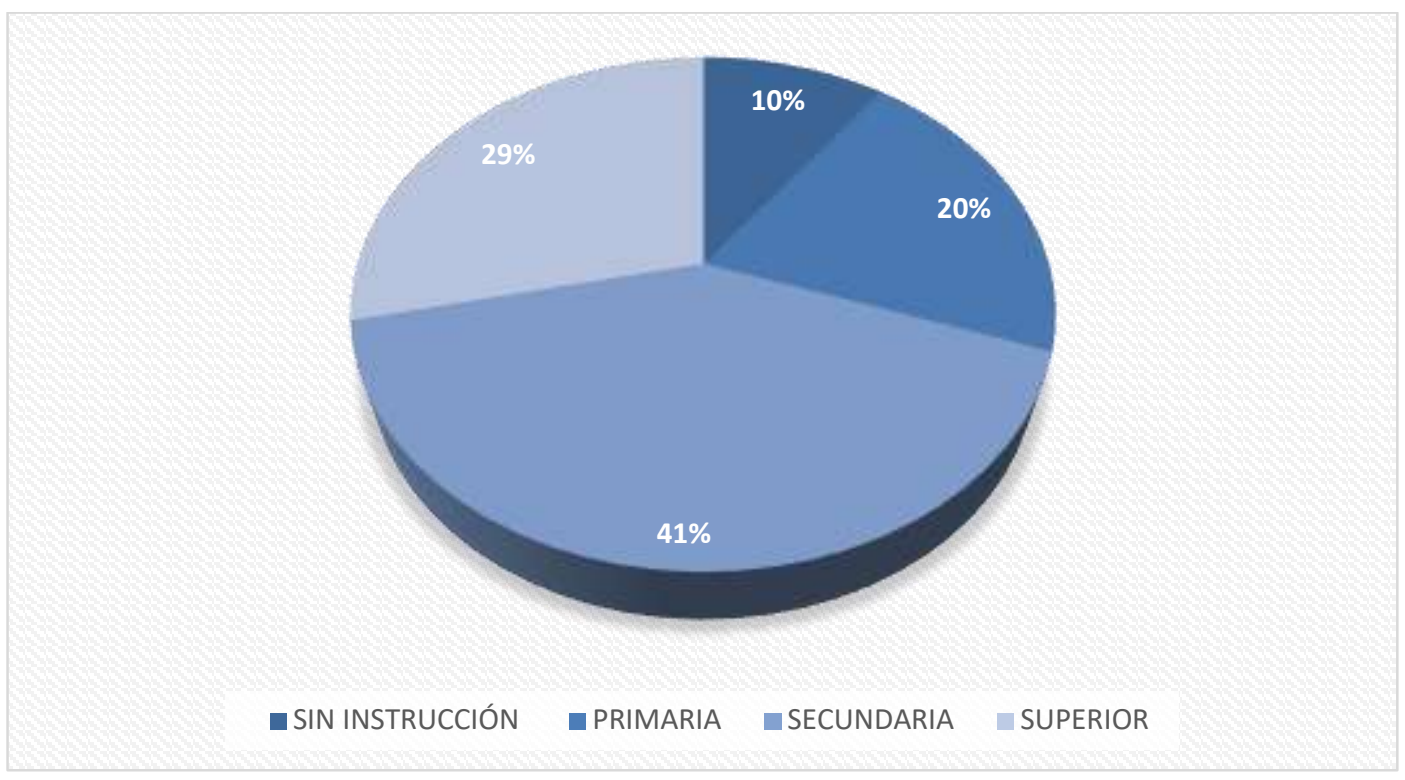

Figura 7. Nivel de formación educacional del contribuyente.

El nivel educativo del contribuyente es importante para poder garantizar el cumplimiento de las obligaciones que tiene la población con el estado, es por eso que analizar las condiciones de estas es relevante, por lo que se estableció los rangos de sin instrucción, primaria, secundaria y superior. Se muestra el mayor porcentaje de encuestados está en la categoría de nivel de educación secundaria en un 41\% (154 personas) y el menor porcentaje de encuestados está en la categoría de sin instrucción educativa con un 10\% (37 personas) (Figura 7). 


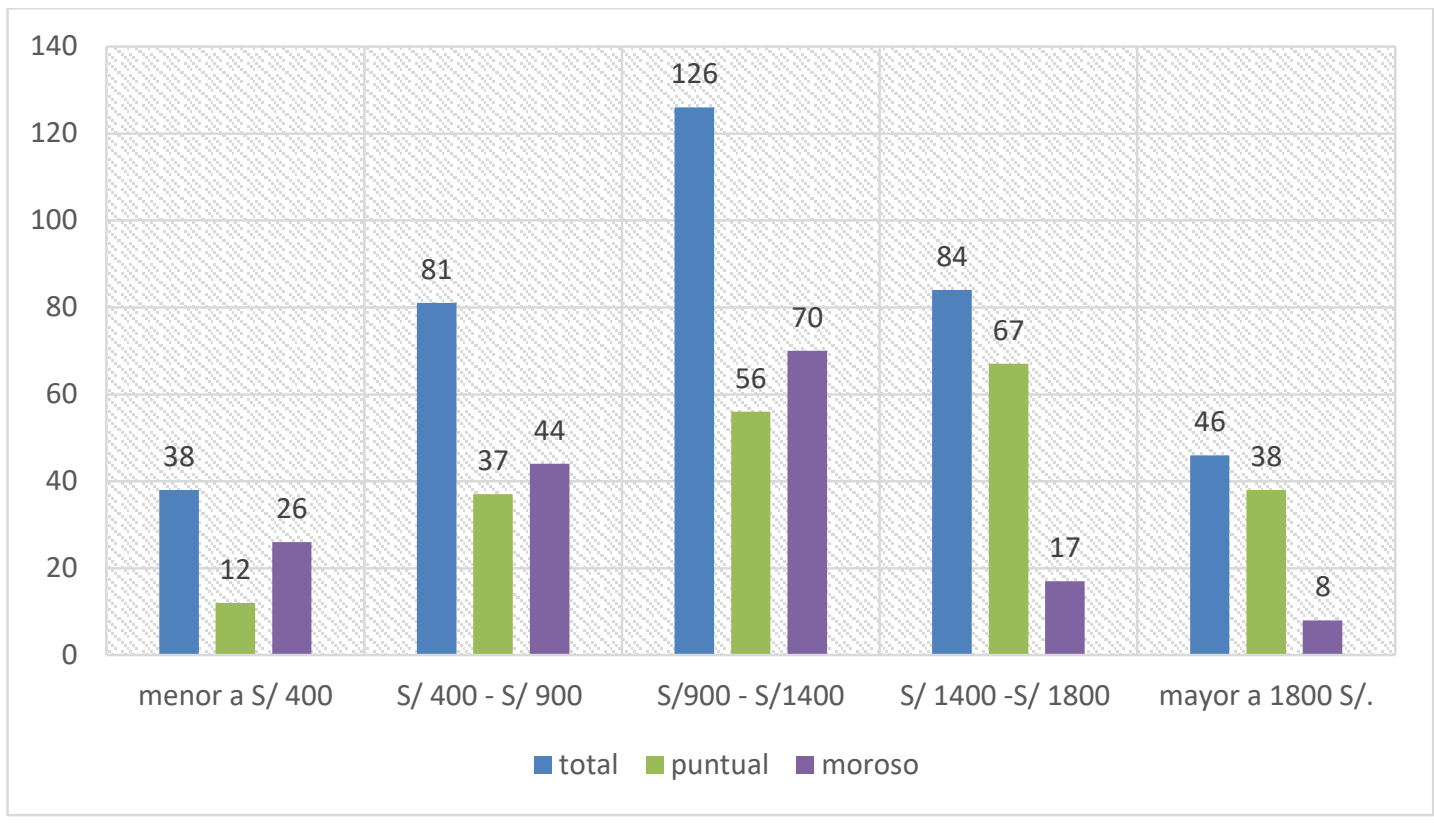

Figura 8. Situación del contribuyente según el ingreso familiar mensual

Finalmente, la otra variable importante es el ingreso familiar mensual que estas perciben, donde se aprecia que del total de encuestados, el grupo que se encuentra con la cantidad de ingresos generados entre S/. 900 - S/. 1,400 soles son los que tienen mayor mora en el pago de los impuestos, es decir pagan a destiempo (Figura 8); por lo que, se evidencia una disminución en la tasa de morosidad cuando las familias tienen mayores ingresos económicos familiares, de esta se cumple la teoría económica de que las familias con menores recursos económicos no tienen la posibilidad de pensar en cubrir otras necesidades u obligaciones que no sean las básicas. Además, se muestra que 26 de las 38 personas que perciben ingreso menor a S/.400 soles, son puntuales con el pago de del Impuesto al Valor del Patrimonio Predial (Figura 8).

\section{Análisis Regresional del modelo Logit Binomial}

Luego de analizar de manera descriptiva las variables de importancia en la presente investigación, es necesario realizar la explicación de estas a través de un modelo logit binomial, ya que, esta permite explicar el comportamiento de las variables que influyen en el cumplimiento del pago del Impuesto al Valor del Patrimonio Predial. 


$$
\operatorname{prob}[Y=1]=F\left(X^{\prime}{ }_{i} \alpha\right)=\frac{e^{X^{\prime}{ }_{i} \alpha}}{1+e^{X^{\prime}{ }_{i} \alpha}}
$$

$\operatorname{prob}[$ cumple $=1]=F\left(X^{\prime}{ }_{i} \alpha\right)$

$$
=\frac{e^{\left.X^{\prime}{ }_{i} \alpha_{0}+\alpha_{1}(\text { cultura tributaria })+\alpha_{2} \text { (ingresofamiliar }\right)+\alpha_{3}(\text { niveleeducativo })}}{1+e^{X^{\prime}{ }_{i} \alpha_{0}+\alpha_{1}(\text { cultura tributaria })+\alpha_{2}(\text { ingresofamiliar })+\alpha_{3}(\text { niveleeducativo })}}
$$

Del cual, los resultados obtenidos en el modelo econométrico logit binomial, ayudo para la explicación de las variables estudiadas esto nos permite interpretar la prueba de hipótesis, en donde estos resultados tienen un nivel de significancia 5\%, trabajando aun nivel de confianza del 95\%. Donde las variables cultura tributaria, ingreso familiar mensual y nivel educativo si tienen una influencia significativa sobre la variable explicada (Tabla 2).

Tabla 2. Resultados de nivel de significancia del modelo

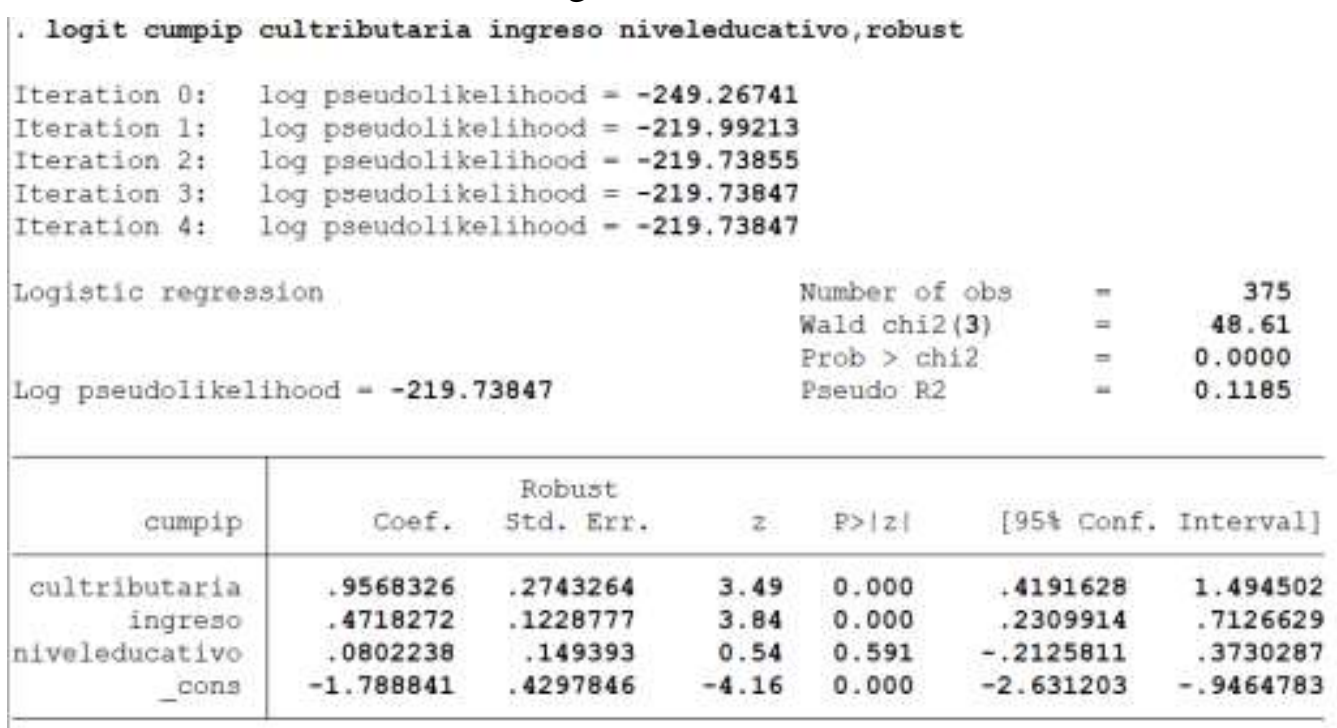

Considerando el Pseudo R2 del modelo, esta nos indica que en conjunto las variables independientes influyen en un $12 \%$ en la variable dependiente. Si el contribuyente recibió alguna vez capacitaciones o talleres de pago de impuestos y tiene conciencia y educación tributaria; es decir, sí tiene cultura tributaria, entonces la probabilidad de que cumpla con el pago del Impuesto al Valor del Patrimonio Predial aumenta (Tabla 2).

Interpretando los efectos marginales obtenidos del modelo se tiene que, la probabilidad en conjunto de las variables independientes que impulsan la probabilidad de que se cumpla con el pago del Impuesto al Valor del Patrimonio Predial es del 63.37\% (Tabla $3)$. 
Si el contribuyente aumenta en $1 \%$ la cantidad de ingreso familiar mensual, entonces la probabilidad de cumplir con el pago de Impuesto al Valor del Patrimonio Predial incrementa en $10.95 \%$ (Tabla 3).

Si el contribuyente percibió al menor un evento de capacitaciones, tiene conciencia y al menor su nivel de formación educacional y en educación tributaria, entonces la probabilidad de que cumpla con el pago del Impuesto al Valor del Patrimonio Predial aumenta en $1.86 \%$ y esto es bastante resaltante (Tabla 3 ).

Si aumenta la cantidad de ingreso familiar mensual generado en $1 \%$, entonces la probabilidad de que el contribuyente cumpla con el pago del Impuesto al Valor del Patrimonio Predial aumenta en $10.95 \%$ (Tabla 3).

Tabla 3. Efectos marginales del modelo

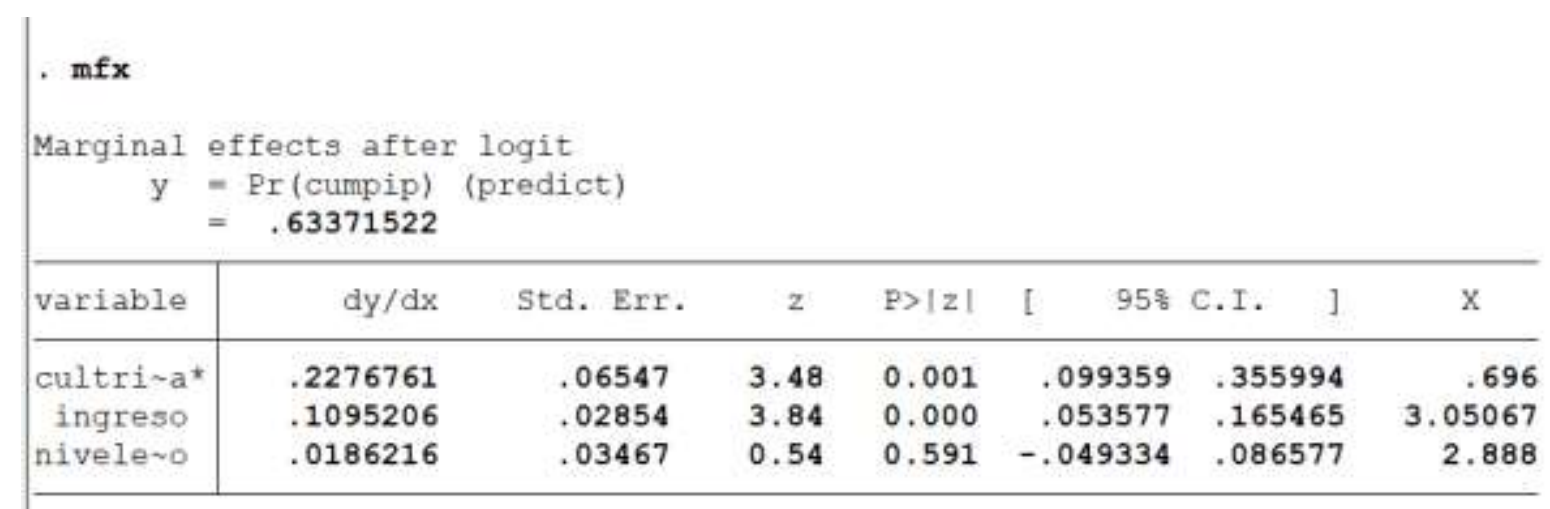

Por lo cual queda demostrado que los factores que influyen en el cumplimiento del pago del Impuesto al Valor del Patrimonio Predial en la ciudad de Puno son la cultura tributaria con $22.77 \%$, nivel educativo con $1.86 \%$ y el ingreso familiar mensual del contribuyente con una probabilidad de $10.95 \%$.

En este sentido, de acuerdo a Esparza (2016), considera que, los contribuyentes no conocen exactamente las obligaciones a las que están afectos, no conocen exactamente la naturaleza de los tributos y su utilización y eso parcialmente se ha demostrado en la presente investigación, en vista que es necesario impulsar el tema de sensibilización y difusión de los objetivos del pago de los impuestos. Al respecto Mostacero \& Belén (2017) menciona que la cultura tributaria no solo es saber cuáles son mis obligaciones como contribuyente, sino que debo aplicar dichos conocimientos en el cumplimiento de 
los deberes tributarios como buen contribuyente y son estos tributos que serán utilizados por el estado en los servicios (Cabana, 2016) que brinda y gastos en beneficio de la población. En los resultados de la presente investigación se demostró que el bajo nivel de cultura tributaria es uno de ellos factores que determinan el bajo nivel de recaudación de Impuesto al Valor del Patrimonio Predial (Caceres, 2018).

Finalmente, al considerar a Rumay \& Angelo (2017), determino que, el nivel de cultura tributaria que existe es bajo, debido a que el $69 \%$ de encuestados respondieron negativamente sobre conocimientos de temas tributarios y sobre la responsabilidad en los pagos (Águila \& Portocarrero, 2013). La presente investigación concuerda con la afirmación mencionada, pues los resultados a los que se llegó nos muestran que el 75\% de los contribuyentes; es decir la mayoría tiene un nivel de cultura tributaria bajo (Agurto, 2018).

\section{CONCLUSIÓN O CONSIDERACIONES FINALES}

Los factores que influyen en el cumplimiento del pago del Impuesto al Valor del Patrimonio Predial en la ciudad de Puno son la cultura tributaria con $22.77 \%$, nivel educativo con $1.86 \%$ y el ingreso familiar mensual del contribuyente con una probabilidad de $10.95 \%$.

Los contribuyentes del Impuesto al Valor del Patrimonio Predial en la ciudad de Puno tienen cultura tributaria, la misma que se encuentra reflejado en un $70 \%$ de la población estudiado; además, el $62 \%$ de los encuestados son conscientes que deben cumplir con el pago de dicho impuesto. El 68\% de los encuestados cuentan con conocimiento acerca de las tasas de contribución municipales, pero a la vez se evidencio que el $78 \%$ de la muestra encuestada nunca recibió algún curso o charlas sobre temas tributarios, o de difusión tributaria.

Finalmente, el ingreso de los contribuyentes es el principal impedimento para la no contribución y ante una política mal empleada, los ingresos de los contribuyentes pueden verse afectado, por lo que en un futuro el incumplimiento podría aumentar. 


\section{LISTA DE REFERENCIAS}

Agresti, A. (2007). An introduction to categorial data analysis. New Jersey: A John Wiley and Sons, Inc.(s.f.).

Águila, A. M., \& Portocarrero, C. S. (2013). factores que influyen en los contribuyentes frente al incumplimiento de pago del impuesto predial en la municipalidad distrital de morales periodo-2013.

Arucutipa, N. M. (2015-2018). Cultura tributaria y nivel de recaudación del impuesto predial en la municipalidad provincial de Yunguyo, periodo 2015-2018.

Calcina, J. A. (2015-2016). La incidencia de la cultura tributaria en la recaudación deel impuesto predial de los contribuyentes de la municipalidad provincial de Azángaro, región Puno 2015, 2016.

Calixto, A. M. (2016). La cultura tributaria y su incidencia en la recaudacion del impuesto predial en la municipalidad provincial de Yarowilca-Huánuco.

Carazas, S. S. (2013,2014). La cultura tributaria como causa del imcumplimiento al pago del impuesto preddial de los contribuyentes de la municipalidad provincial de Ayaviri periodos 2013,2014.

Cobeñas, D. A., Jacinto, L. G., \& Quinde, F. A. (octubre de 2018). LA CULTURA TRIBUTARIA Y SU RELACIÓN CON EL PAGO DEL IMPUESTO AL VALOR DEL PATRIMONIO PREDIAL EN EL DISTRITO DE LA UNIÓN. PIURA-PERÚ.

Obtenido

de http://premio.investiga.fca.unam.mx/docs/ponencias/2018/6.04.pdf

Cueva, Y. M., \& Serrano, J. A. (2017). La cultura tributaria y su influencia en la recaudación del impuesto predial en la municipalidad distrital de Namora, 2017.

Garay, F. E. (2017). La conciencia tributaria y su influencia en la evasión tributaria del impuesto predial en la municipalidad distrital de Santa María, 2017. 
Huarilloclla, R. C. (2015). La cultura trbutaria y su influencia en el cumplimiento de las obligaciones tributarias de los comerciantes del mercado central del distrito de Santa Lucia, periodo 2015.

Huisa, B. Y. (2014-2015). Cultura tributaria y su incidenncia en la recaudación del impuesto predial de la municipalidad pronvincial de Puno, periodos 2014_2015.

Lujano, K. K. (2016). “DETERMINACIÓN DE LA EVASIÓN TRIBUTARIA Y SU INCIDENCIA EN LA RECAUDACIÓN DEL IMPUESTO PREDIAL EN LA MUNICIPALIDAD PROVINCIAL DE PUNO PERIODOS 2013, 2014 Y 2015” Obtenido de http://repositorio.unap.edu.pe/bitstream/handle/UNAP/3902/Flores_Lujano_Kar em_Katherine.pdf?sequence $=1 \&$ isAllowed $=\mathrm{y}$

Mamani, J. R. (2014). La incidencia de la cultura tributaria en la recaudación del impuesto predial de los contribuyentes de la municipalidad provincial de Lampa, region Puno 2014. PUNO.

Massimino, L. F. (2015). La intervención estatal, la regulación económica y el poder de policía: análisis y tendencias. DIREITO ECONÓMICO E SOCIOAMBIENTAL.

Mostacero, L. B. (2016). Incidencia de la cultura tributaria municipal en la declacion jurada del impuesto predial de los contribuyentes del servicio de administración triibutaria de Trujillo (SATT), 2016.

MUNICIPALIDAD PROVINCIAL DE PUNO. (s.f.). Obtenido de http://www.munipuno.gob.pe/tributos_municipales/predios.htm

Perdomo, T. V., \& Egáñez, M. T. (2009). CONOCIMIENTO Y EDUCACION EN EL CONTRIBUYENTE VENEZOLANO SOBRE LA CULTURA TRIBUTARIA. DAENA.

Perez, C. P. (2008). SOBRE EL CONCEPTO DE VALOR. UNA PROPUESTA DE INTEGRACION DE DIFERENTES PERSPECTIVAS.

Rojas, A. M. (2018). Cultura tributaria y la recaudación del impuesto predial en la municipalidad distrital de el Tambo,2018. 
Rumay, D. A. (2016). La cultura tributaria y su efecto en la recaudación del impuesto predial en la municipalidad distrital de Chocope, 2016.

Sampieri, R. H., Collado, C. F., \& Lucio, P. B. (2010). Metodologia de la investigación. México: Miembro de la Cámara Nacional de la Industria Editorial Mexicana.

Samuelson, P. A. (1999). Economia . España: Mc graw-hill interamericano de España s.au.

SUPERINTERDENCIA DE ADMINISTRACIÓN TRIBUTARIA. (s.f.). SAT VIRTUAL. Obtenido de https://portal.sat.gob.gt/portal/cultura-tributaria/

Zegovia, J. M. (2014). cumplimiento de la obligacion tributaria del impuesto predial y su relación con el nivel de recaudación fiscla en la municipalidad distrital Alto de la Alianza, año 2014. 